\title{
Sonographic normal values for the cross-sectional area of the ulnar nerve: a systematic review and meta-analysis
}

\author{
Nadine Boers ${ }^{1,5}$ - Enrico Martin ${ }^{1} \cdot$ Marc Mazur $^{1}$ - David D. Krijgh ${ }^{1} \cdot$ Monique H. M. Vlak ${ }^{2}$. Godard C. W. de Ruiter ${ }^{3}$. \\ H. Stephan Goedee ${ }^{4} \cdot$ J. Henk Coert ${ }^{1}$
}

Received: 9 November 2021 / Accepted: 14 January 2022 / Published online: 19 February 2022

(c) The Author(s) 2022

\begin{abstract}
Purpose Nerve size is a commonly used sonographic parameter when assessing suspected entrapment of the ulnar nerve. We aimed to create a robust set of normal values, based on a critical review of published normal values.

Methods We performed a systematic evaluation of studies on normal ulnar nerve sizes, identified in PubMed, Embase, and Cochrane databases. Using meta-analyses, we determined pooled mean cross-sectional area (CSA) values for different anatomical locations of the ulnar nerve throughout the arm. Subgroup analyses were performed for gender, probe frequency, in- or exclusion of diabetic patients, position of the elbow and Asian versus other populations.

Results We identified 90 studies of which 77 studies were included for further analyses after quality review, resulting in data from 5772 arms of 3472 participants. Subgroup analyses show lower CSA values at at the wrist crease and proximal to the wrist crease when using low frequency probes $(<15 \mathrm{MHz})$ and at the wrist crease, proximal to the wrist crease, proximal forearm and the distal upper arm in Asians. CSA values were lower when in flexed position compared to extended position for the cubital tunnel inlet only. No difference was found for gender.

Conclusions Our systematic review provides a comprehensive set of normal values at sites along the entire length of the ulnar nerve. This provides a foundation for clinical practise and upon which future studies could be more systematically compared.
\end{abstract}

Keywords Ultrasound $\cdot$ Ulnar nerve $\cdot$ Cross-sectional area $\cdot$ Reference values $\cdot$ Normal values

\section{Introduction}

Ultrasound is increasingly used in the diagnosis of neuropathies, including ulnar nerve entrapment, and importantly complementing electrodiagnostic studies. As point of care device, advantages of nerve ultrasound include low cost,

Nadine Boers

n.boers-2@umcutrecht.nl; nadine95boers@hotmail.com

1 Department of Plastic Surgery, Utrecht Medical Center, Utrecht, The Netherlands

2 Department of Neurology, Haaglanden Medical Center, The Hague, The Netherlands

3 Department of Neurosurgery, Haaglanden Medical Center, The Hague, The Netherlands

4 Department of Neurology, Utrecht Medical Center, Utrecht, The Netherlands

5 Department of Plastic, Reconstructive, and Hand Surgery, University Medical Center Utrecht, Heidelberglaan 100, 3584 CX Utrecht, The Netherlands practical non-invasive bedside testing, wide availability and flexible field of view [1]. It can provide essential diagnostic information on relevant morphological changes, such as precise anatomical site and exact nature [2-4]. Several sonographic findings have been proposed to aid in diagnosis of ulnar nerve entrapment: swollen segment surrounding the nerve, increased intraneural vascularity, reduced mobility, blurred margins, and a loss of the fascicular pattern [5]. At present, the nerve size, expressed in cross-sectional area (CSA), appears to be the most robust sonographic parameter. An expert panel recently agreed that ultrasound of the ulnar nerve should include assessment of the CSA and nerve mobility at the elbow and that the entire ulnar nerve from wrist to axilla should be imaged [6].

Unfortunately, previous studies used diverse ultrasound protocols in evaluation of ulnar neuropathy. Consequently, diagnostic cut-off values for CSA measurements vary between studies, resulting in different sensitivities and specificities [3]. Besides, most of the reported variability in literature stem from a lack of standardized normal values 
for the ulnar nerve. In diagnostic accuracy studies, control groups are often small, comparable to the size of the symptomatic group. The few studies that provide reference values ha for only four locations of the ulnar nerve [7]. Adding data from control groups makes it possible to create reference values at more locations along the ulnar nerve. Therefore, we aimed to systematically evaluate published data on sonographic normal values for ulnar nerve size to create a robust set of reference values which can be used in clinical and research settings.

\section{Methods}

We performed a review in accordance to the PRISMA (Preferred Reporting Items for Systematic Reviews and MetaAnalysis) guidelines, registered in PROSPERO, the International Prospective Register of Systematic Reviews before conduction (registration number: CRD42021232492) [8].

\section{Search strategy}

We performed databases searches on January 21st 2021 in PubMed, Embase, and Cochrane Library using synonyms, key terms, and MeSH descriptors or Emtree terms for the following words: 'ulnar', 'ultrasound', 'cross-sectional area', and 'reference'. Conference abstracts were excluded. Full search strategies for all three databases are shown in Online Supplementary File A, Table 1. After screening of titles and abstracts, we screened full-text articles for final eligibility. We searched trough reference for additional papers. Screening was performed by two independent researchers (N.B. and M.M.) and discrepancies in selected papers were discussed with a third reviewer (S.G.) until consensus was reached.

\section{Study selection}

We included studies when the following criteria were met: (1) direct CSA measurements of the ulnar nerve were performed in healthy participants; (2) clear description of the study population and sonographic protocol for image acquisition, including the anatomic locations of the ulnar nerve measurements. Exclusion criteria were lack of full text, case reports, conference abstracts, reviews and language other than English.

\section{Data extraction}

Two independent researchers (N.B. and M.M.) extracted study, patient, and ultrasound characteristics from included studies and noted mean or median CSA values with $95 \%$ confidence interval (CI), standard deviation (SD) and/or range for all available anatomic locations. We categorized the anatomic locations into 11 categories, as shown in Fig. 1. Figure 2 shows an example of the ulnar nerve and its CSA measurement at the cubital tunnel, a possible point of compression.

For each study we also extracted data on size of study population, whether or not patients with diabetes were excluded, demographic data, number and side of arms studied, the type of probe $(<15 \mathrm{mHz}$ or $>15 \mathrm{mHz})$ and the position of the elbow at investigation (extended, flexed or unknown).

We contacted twenty authors by email when relevant information on patient characteristics, methods or outcomes were missing. After reminder mails, we received additional information from six authors on six studies.

\section{Quality assessment}

Two in-dependent researchers (N.B. and M.M.) appraised the quality of included studies using the Quality Assessment of Diagnostic Accuracy Studies (QUADAS-2) tool, designed to assess the quality of primary diagnostic accuracy studies (Table 2, Online Supplementary File A) [9]. Authors solved disagreement trough discussion. Studies have a high risk of bias when diabetic patients were not specifically excluded, when radiologists were not blinded, when data was collected during different periods and in different settings or when not all patients were included in analyses. Authors had applicability concerns when study conduction or study population was not representative for the research question of the study. When assessing the overall risk of bias, authors did not take the domain 'reference standard' into account as we are only interested in data on healthy participants. The Risk of Bias Table and Graphs summarize the quality appraisal for all studies included in the quantitative analyses.

\section{Statistical analysis}

We were able to include studies in quantitative analyses if they reported mean values with SD or 95\% CI's. Using the formulas recommended by to the Cochrane Handbook, we calculated SD values from 95\% CI's [10]. We did not calculate SD values from ranges as this is not recommended.

We performed random effects meta-analyses of mean CSA values with SD when at least three studies reported CSA values. For the main analyses, we included data from both female and male and right and left arm. If papers only displayed subgroup data (i.e. female and male or right and left side), we used pooled mean values. When studies used overlapping data, we selected data from the largest and most appropriate study for inclusion in quantitative synthesis. If studies presented separate CSA values for flexion and extension of the elbow, we used CSA values of flexed elbows in the main analyses as this was considered 


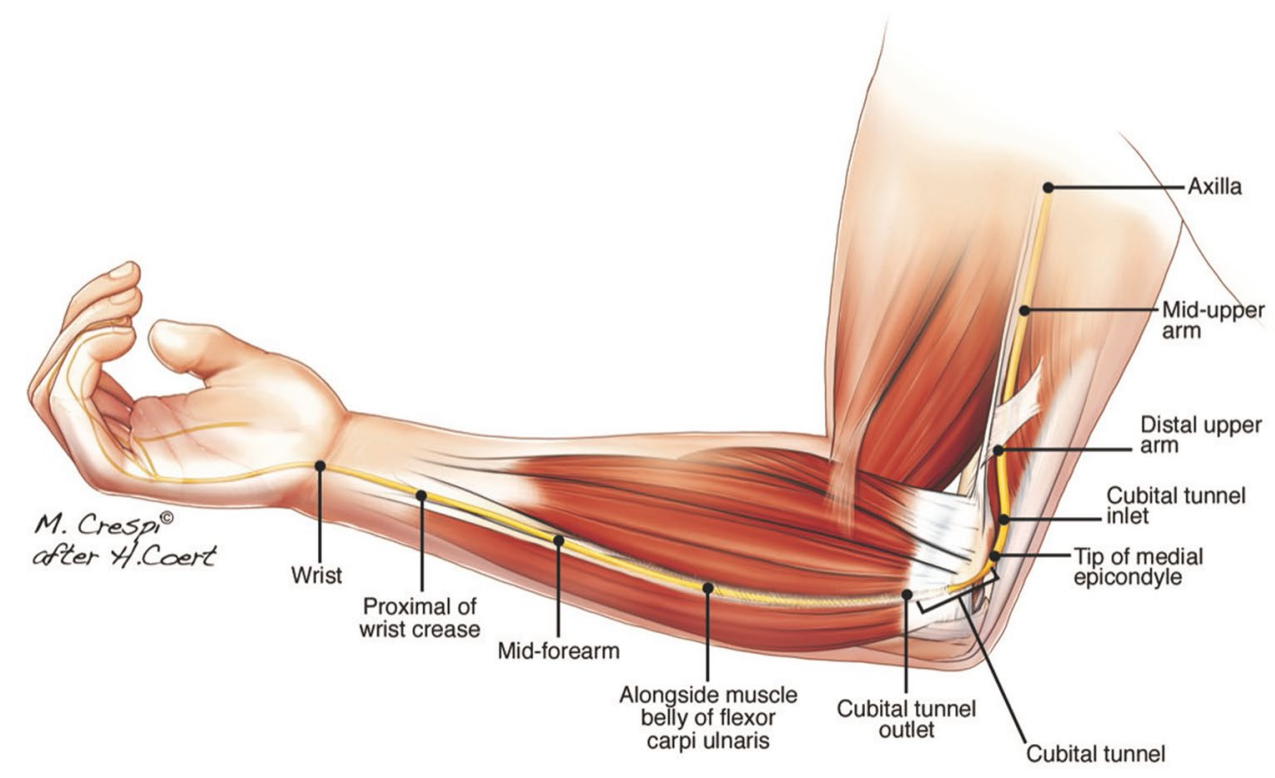

Fig. 1 Anatomical landmarks. 11 anatomical locations: the wrist crease (the distal wrist crease and Guyon's canal), proximal of wrist crease (from $2 \mathrm{~cm}$ proximal to distal crease to distal $1 / 3 \mathrm{rd}$ of the forearm), mid-forearm (where the ulnar artery and nerve make contact), proximal forearm ( $2 \mathrm{~cm}$ proximal to the contact point of ulnar artery and nerve to $3 \mathrm{~cm}$ distal to tip of the medial epicondyle), cubital tunnel outlet $(1-2 \mathrm{~cm}$ distal to medial epicondyle, between the two heads of the flexor carpi ulnaris muscle), cubital tunnel (maximal CSA measurements between cubital tunnel inlet and outlet), tip of the medial epicondyle, cubital tunnel inlet $(1-2 \mathrm{~cm}$ proximal to medial epicondyle), distal upper arm (4-5 $\mathrm{cm}$ proximal to tip of the medial epicondyle), mid-upper arm and axilla. The authors hold the copyright for this figure as it was made specifically for this article

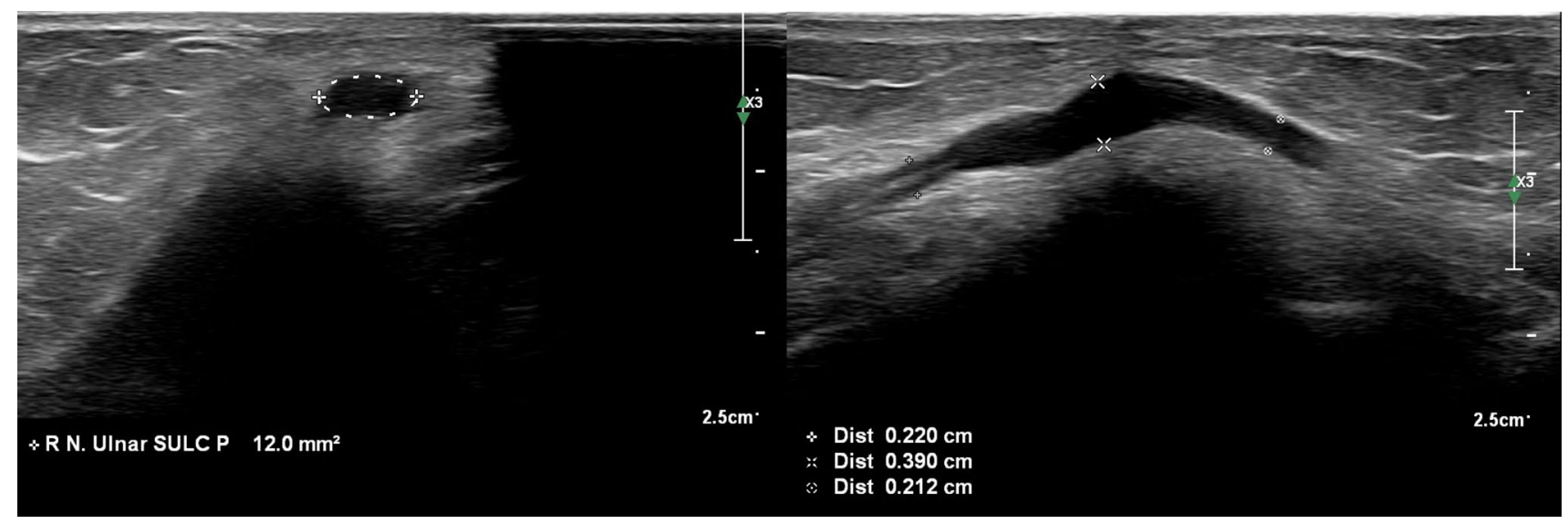

Fig. 2 Ultrasound image of the ulnar nerve at the cubital tunnel. Left: a cross-sectional view of the ulnar nerve at the cubital tunnel. Right: a longitudinal view of the ulnar nerve at the cubital tunnel where thickening of the nerve is shown

the standard position and was used in most studies. The $I^{2}$ test statistic was used to evaluate heterogeneity of CSA means across studies. $P<0.1$ or $I^{2}>50 \%$ was classified as a high degree of heterogeneity among studies.

Subgroup analyses were performed for studies in which (1) men and women were reported separately; (2) probe frequency was noted $[<15 \mathrm{MHz}$ (low resolution) or $\geq 15 \mathrm{MHz}$ (high resolution)]; (3) explicitly reported excluding diabetic patients versus studies who did not report this; (4) flexion of the elbow versus extension of the elbow, and (5) Asian versus other populations. We performed subgroup analyses only when subgroups included at least three studies.

All statistical analyses were performed with $\mathrm{R}$ version 4.0.2 (R Core Team, 2021). 


\section{Results}

\section{Search results and study characteristics}

Figure 3 summarizes our literature search and study selection. We found 90 studies that met the inclusion criteria, including 6806 elbows of 4206 participants (Online Supplementary File B). Of these, 56 studies compared disease specific CSA values to a control group, and 34 studies included healthy participants with the main goal to determine reference values. Study characteristics for all included studies are shown in Online Supplementary File C, Table 4. The reported CSA values for all included studies are summarized in Online Supplementary File C, Table 5 (adults), Table 6 (children), Table 7 (gender subgroups) and Table 8 (age subgroups).

\section{Meta-analysis}

We included 77 studies for quantitative analyses, including 5772 elbows of 3472 participants. Of these studies, 44 studies had high overall risk of bias, mainly due to not specifically excluding diabetic patients and lack of examiner blinding. The Risk of Bias Graph including outcomes for each domain are shown in Online Supplementary File C, Table 3 and Fig. 1. We found a high degree of heterogeneity between studies in all meta-analyses, including subgroups. Table 1

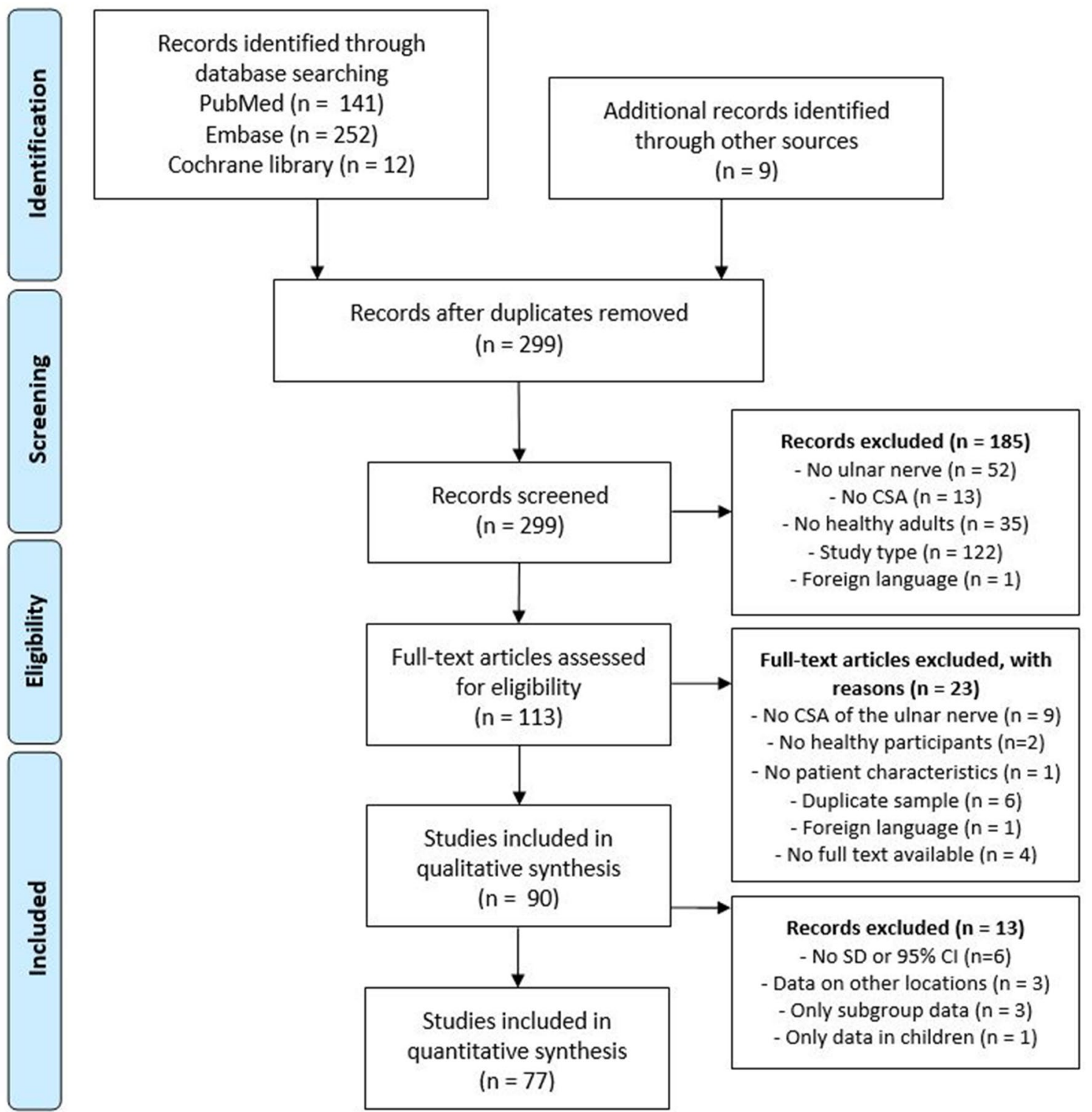

Fig. 3 PRISMA flow diagram 
Table 1 Pooled CSA stratified for anatomical level of the ulnar nerve

\begin{tabular}{|c|c|c|c|c|}
\hline Anatomical location & $\mathrm{N}$ studies & N elbows & CSA $(95 \% \mathrm{Cl})$ & Forest plot \\
\hline Wrist crease & 33 & 2311 & $4.84(4.47-5.21)$ & $\rightarrow$ \\
\hline Proximal of wrist crease & 10 & 942 & $5.37(4.57-6.17)$ & $\rightarrow-$ \\
\hline Mid-forearm & 34 & 2218 & $5.78(5.45-6.11)$ & $\rightarrow$ \\
\hline Proximal forearm & 8 & 588 & $5.03(4.58-5.48)$ & $\rightarrow$ \\
\hline Cubital tunnel outlet & 17 & 1283 & $6.18(5.45-6.90)$ & $\rightarrow$ \\
\hline Tip of medial epicondyle & 36 & 2529 & $6.81(6.31-7.31)$ & $\rightarrow$ \\
\hline Cubital tunnel & 15 & 1211 & $6.50(5.80-7.20)$ & $\rightarrow$ \\
\hline Cubital tunnel inlet & 19 & 1452 & $6.81(6.26-7.36)$ & $\rightarrow$ \\
\hline Distal upper arm & 8 & 670 & $4.67(4.39-4.94)$ & $\rightarrow$ \\
\hline Mid-upper arm & 25 & 1923 & $6.19(5.73-6.66)$ & $\rightarrow$ \\
\hline Axilla & 4 & 632 & $5.37(4.57-6.16)$ & $\longrightarrow$ \\
\hline
\end{tabular}

Includes all studies included in quantitative analyses. Included studies and according references are shown in the appendix. $95 \%-\mathrm{Cl}=95 \%$ confidence interval of the mean CSA value; $\mathrm{n}=$ number.

summarizes the pooled mean CSA values of the ulnar nerve, stratified for anatomical location. Online Supplementary File C, Fig. 2 shows forest plots of pooled mean CSA values.

\section{Subgroup analyses}

Pooled CSA values were lower for subgroups with a low frequency probe (range $<15 \mathrm{MHz}$ ) compared to high frequency probe (range $\geq 15 \mathrm{MHz}$ ) at the wrist crease and proximal to the wrist crease. CSA values were lower for the Asian population compared to the non-Asian population at the wrist crease, proximal to the wrist crease, proximal forearm and the distal upper arm. Only for the cubital tunnel inlet, CSA values were lower with the elbow in flexed position compared to extended position. We found no difference for gender and between studies explicitly excluding diabetes patients and those who did not. Statistically significant different pooled CSA values for the subgroup analyses are shown in Table 2. An overview of all subgroup analyses and corresponding forest plots are shown in Online Supplementary File C, Table 9 and Fig. 3.

\section{Branches of the ulnar nerve in the hand}

CSA values of branches of the ulnar nerve (i.e. deep branch of the ulnar nerve, superficial branch of the ulnar nerve, dorsal ulnar cutaneous nerve and palmar ulnar cutaneous nerve) in the hand were described in only four studies and are shown in Table C.2 (Online Supplementary File C) [11-14].

\section{Discussion}

Our meta-analyses resulted in a robust set of normal values specific anatomical sites along the entire length of the ulnar nerve. We found lower CSA values in low resolution probes $(<15 \mathrm{MHz})$ and with $>90^{\circ}$ flexion of the elbow. Also we found lower CSA values in the Asian population, which therefore may warrant modified reference values. No difference was found for gender.

Diagnostic ultrasound of the ulnar nerve often relies on evaluation of nerve size, commonly rated as CSA on transverse images. Several other sonographic parameters have been proposed to be of potential additional value, however, the number of studies on these are still limited. Experts recently agreed that not only the elbow, but the entire length of the ulnar nerve should be imaged [6]. Therefore, our meta-analyses focused on CSA values for unique anatomical sites along the length of the ulnar nerve.

We have identified and analysed different equipment and techniques, including different probe frequencies (<12 MHz, 12-15 MHZ, 15-18 MHz or a broader range), tracing methods (automatic tracing, free-hand tracing, ellipsoid function or irregular tracing according to the nerve shape), scan protocols, position of the arm $\left(90^{\circ}\right.$ flexion in the elbow, $>90^{\circ}$ flexion, $<90^{\circ}$ flexion, extension or not described), and blinding of the ultrasonographer. Deploying ultrasound probes with lower frequencies warrant caution as image resolution is lower and consequently, the nerve boundaries are less sharp, thus making it more difficult to accurately measure the nerve [15]. Consequently, our subgroup analyses, showed lower pooled CSA values of the ulnar nerve at, and proximal to, the wrist in studies using a 
Table 2 Subgroup analyses with significantly different pooled CSA values

\begin{tabular}{|c|c|c|c|c|c|}
\hline Position & Subgroup & & $N$ of studies & Mean CSA $(95 \%$ CI $)$ & $p$-value \\
\hline \multirow[t]{4}{*}{ Wrist crease } & \multirow[t]{2}{*}{$\mathrm{Hz}$} & $<15 \mathrm{MHz}$ & 18 & $4.56(4.08-5.03)$ & \multirow[t]{2}{*}{0.048} \\
\hline & & $\geq 15 \mathrm{MHz}$ & 15 & $5.17(4.79-5.55)$ & \\
\hline & \multirow[t]{2}{*}{ Asian } & Asian population & 14 & $4.25(3.75-4.74)$ & \multirow[t]{2}{*}{0.001} \\
\hline & & Other & 19 & $5.26(4.91-5.61)$ & \\
\hline \multirow[t]{4}{*}{ Proximal of wrist crease } & \multirow[t]{2}{*}{$\mathrm{Hz}$} & $<15 \mathrm{MHz}$ & 6 & $4.74(4.03-5.44)$ & \multirow[t]{2}{*}{0.004} \\
\hline & & $\geq 15 \mathrm{MHz}$ & 3 & $6.67(5.57-7.77)$ & \\
\hline & \multirow[t]{2}{*}{ Asian } & Asian population & 6 & $4.74(4.03-5.44)$ & \multirow[t]{2}{*}{0.004} \\
\hline & & Other & 3 & $6.67(5.57-7.77)$ & \\
\hline \multirow[t]{2}{*}{ Proximal forearm } & \multirow[t]{2}{*}{ Asian } & Asian population & 3 & $4.42(4.06-4.77)$ & \multirow[t]{2}{*}{0.006} \\
\hline & & Other & 5 & $5.42(4.80-6.04)$ & \\
\hline \multirow[t]{3}{*}{ Cubital tunnel inlet } & \multirow[t]{3}{*}{ Position } & Flexion & 9 & $7.25(6.70-7.80)$ & \multirow[t]{3}{*}{0.003} \\
\hline & & Extension & 3 & $6.09(5.72-6.45)$ & \\
\hline & & Unknown & 7 & $6.48(5.45-7.51)$ & \\
\hline \multirow[t]{3}{*}{ Distal upper arm } & \multirow[t]{3}{*}{ Asian } & Asian population & 4 & $4.30(4.07-4.53)$ & \multirow[t]{3}{*}{0.029} \\
\hline & & Other & 4 & $4.87(4.42-5.32)$ & \\
\hline & & Male & 4 & $5.69(4.85-6.52)$ & \\
\hline
\end{tabular}

Only subgroup analyses with p-values below 0.05 are shown

95\% CI 95\% confidence interval of the mean CSA value, CSA cross-sectional area, $n$ number. $p$ values $<0.05$ were considered significant lower probe frequency ( $<15 \mathrm{MHz}$ versus $\geq 15 \mathrm{MHz}$ ). Previous data on zoom magnification, affecting the CSA values, indicated that (lower) resolution should be taken into account when assessing sonographic nerve size [16]. The fact that CSA values decrease with a distal gradient may further compound this. Regarding the position, flexion of the elbow at $90^{\circ}$ was considered the standard position and was used in most studies. However, flexion ranged from $40^{\circ}$ to $135^{\circ}$ and some studies performed measurement with the elbow in extension. As peripheral nerves are capable of stretch and deformation, the position of the upper limb is reported to influence the geometric shape and position of the ulnar nerve [17-21]. Kutlay and Roodt et al. reported a higher CSA in extended upper limbs, contrasting our finding of a higher pooled CSA at the cubital tunnel inlet with the elbow in flexed position [17, 22].

Besides these technical aspects, patient characteristics such as ethnicity may also influence CSA values. We found studies on Asian populations to report a lower CSA at several anatomical sites. A plausible explanation could be racial and ethnic differences among the Asian population compared to non-Asian, or a possibly lower BMI [23]. This is in line with the study of Walhout-van Burg et al. who found that CSA reference values for the median nerve were significantly lower in Asian subjects compared to Dutch people, even after correcting for age, height, and weight [24]. Another explanation could be that studies on Asian populations used lower $\mathrm{Hz}$ frequencies more often $(81 \%$ in Asian studies versus $59 \%$ in all studies included in the metaanalyses). However, we were not able to conduct subgroup analyses on use of lower/higher $\mathrm{Hz}$ frequencies within studies on Asian populations since too little studies used a higher frequency.

BMI has been suggested to influence the CSA value as well. A study comparing American and European CSA values of the median nerve found significantly higher CSA values in the American cohort, possibly explained by the generally higher BMI in the American population [25]. In our study, only three American studies were included in the meta-analyses in which CSA values were mostly comparable or a little higher than in other studies [26-28]. Only one study, including extremes of BMI (mean BMI of $35.2 \mathrm{~kg}$ / $\mathrm{m}^{2}$ ), had noticeably higher CSA values, supporting the hypothesis that BMI may influence the CSA value. One of the included studies compared CSA values of the ulnar nerve between subgroups based on BMI $\left(<25 \mathrm{~kg} / \mathrm{m}^{2}\right.$ versus $\geq 25 \mathrm{~kg} / \mathrm{m}^{2}$ ) and found no difference at the wrist and in the hand [14]. However, included participants all had a BMI below $30.0 \mathrm{~kg} / \mathrm{m}^{2}$ and do not represent extremes of BMI.

Our study has several limitations. We were not able to include all studies in the quantitative analyses because of reported median values or missing SD or $95 \%$ CI values. However, as these included only few studies and raw data shows that these values are not outliers, we feel it is unlikely that this may have influenced our pooled CSA values. Also, of the identified studies, $13 \%$ had unknown and $59 \%$ had high risk of bias. This was mostly due to patient selection as they did not specifically (report to) exclude diabetic patients. Since our primary aim is to obtain reference values, ideally, diabetic patients are excluded from analyses. Patient with 
diabetes may harbor larger CSA values, as has been suggested by previous studies that found higher values in carpal tunnel syndrome without diabetic neuropathy, and the ulnar nerve in (subclinical) diabetic peripheral neuropathy [23, 29-31]. However, we expect that the amount of diabetics included is negligible and therefore, is not likely to have influenced our pooled CSA values.

\section{Conclusion}

We provided a robust set of normal values at several anatomical sites of the ulnar nerve that can be used in diagnostic and research settings. Our study found lower CSA values in Asian populations which therefor may need modified reference values. Our findings also indicate that the diverse techniques used to perform ultrasound warrant further standardisation of the diagnostic ultrasound procedures, including CSA measurements, to avoid unwanted variation in diagnostic performance and improve comparison in future research.

Supplementary Information The online version contains supplementary material available at https://doi.org/10.1007/s40477-022-00661-8.

Acknowledgements We would kindly like to thank dr. S.M. Pompe, dr. L.H. Visser, dr. A. Volpe, dr. B. Noszczyk, dr. M.E. Ghanei and prof. dr. A. Grimm for providing additional patient information or data on outcomes to be included in this meta-analysis.

Funding There was no funding for this study.

\section{Declarations}

Conflict of interest The authors have no relevant financial or non-financial interests to disclose.

Open Access This article is licensed under a Creative Commons Attribution 4.0 International License, which permits use, sharing, adaptation, distribution and reproduction in any medium or format, as long as you give appropriate credit to the original author(s) and the source, provide a link to the Creative Commons licence, and indicate if changes were made. The images or other third party material in this article are included in the article's Creative Commons licence, unless indicated otherwise in a credit line to the material. If material is not included in the article's Creative Commons licence and your intended use is not permitted by statutory regulation or exceeds the permitted use, you will need to obtain permission directly from the copyright holder. To view a copy of this licence, visit http://creativecommons.org/licenses/by/4.0/.

\section{References}

1. Mezian K, Jačisko J, Kaiser R et al (2021) Ulnar neuropathy at the elbow: from ultrasound scanning to treatment. Front Neurol. https://doi.org/10.3389/FNEUR.2021.661441
2. de Ruiter GCW, de Jonge JGH, Vlak MHM, van Loon-Felter AE (2020) Ulnar neuropathy caused by muscular arcade of struthers. World Neurosurg. 142:128-130

3. Chang K-V, Wu W-T, Han D-S, Özçakar L (2018) Ulnar nerve cross-sectional area for the diagnosis of cubital tunnel syndrome: a meta-analysis of ultrasonographic measurements. Arch Phys Med Rehabil 99:743-757. https://doi.org/10.1016/j.apmr.2017. 08.467

4. Van Den Berg PJ, Pompe SM, Beekman R, Visser LH (2013) Sonographic incidence of ulnar nerve (sub)luxation and its associated clinical and electrodiagnostic characteristics. Muscle Nerve 47:849-855. https://doi.org/10.1002/mus.23715

5. London ZN, Burke JF, Hazan R et al (2014) Electromyographyrelated pain: muscle selection is the key modifiable study characteristic. Muscle Nerve 49:570-574. https://doi.org/10.1002/mus. 23974

6. Pelosi L, Arányi Z, Beekman R et al (2021) Expert consensus on the combined investigation of ulnar neuropathy at the elbow using electrodiagnostic tests and nerve ultrasound. Clin Neurophysiol. https://doi.org/10.1016/J.CLINPH.2021.04.018

7. Fisse A, Katsanos A, Gold R et al (2021) Cross-sectional area reference values for peripheral nerve ultrasound in adults: a systematic review and meta-analysis-Part I: upper extremity nerves. Eur J Neurol 28:1684-1691. https://doi.org/10.1111/ENE.14759

8. Page MJ, McKenzie JE, Bossuyt PM et al (2021) The PRISMA 2020 statement: an updated guideline for reporting systematic reviews. Syst Rev. https://doi.org/10.1186/s13643-021-01626-4

9. Whiting PF, Rutjes AWS, Westwood ME et al (2011) Quadas-2: a revised tool for the quality assessment of diagnostic accuracy studies. Ann Intern Med 155:529-536

10. Higgins J, Thomas J, Chandler J et al (2021) Cochrane Handbook for Systematic Reviews of Interventions version 6.2 (updated February 2021). In: Cochrane. www.training.cochrane.org/handbook. Accessed 20 Dec 2020

11. Kim KH, Lee SJ, Park BK, Kim DH (2018) Sonoanatomy of sensory branches of the ulnar nerve below the elbow in healthy subjects. Muscle Nerve 57:569-573. https://doi.org/10.1002/mus. 25959

12. Le Corroller T, Bauones S, Acid S, Champsaur P (2013) Anatomical study of the dorsal cutaneous branch of the ulnar nerve using ultrasound. Eur Radiol 23:2246-2251. https://doi.org/10.1007/ s00330-013-2832-z

13. Riegler G, Lieba-Samal D, Brugger PC et al (2017) High-resolution ultrasound visualization of the deep branch of the ulnar nerve. Muscle Nerve 56:1101-1107. https://doi.org/10.1002/mus.25614

14. Choi H, Yoon JS (2020) Sonoanatomy of the deep branch of the ulnar nerve. Muscle Nerve 61:504-507. https://doi.org/10.1002/ mus. 26820

15. Hedrik W, Hykes D, Starchman D (1995) Ultrasound Physics and instrumentation, 3rd edn. St Louis, Mosby

16. Jelsing E, Presley J, Maida E et al (2015) The effect of magnification on sonographically measured nerve cross-sectional area. Muscle Nerve 51:30-34. https://doi.org/10.1002/MUS.24274

17. Kutlay M, Colak A, Simşek H et al (2009) Use of ultrasonography in ulnar nerve entrapment surgery-a prospective study. Neurosurg Rev 32:225-232. https://doi.org/10.1007/s10143-008-0162-4

18. Gelberman RH, Yamaguchi K, Hollstien SB et al (1998) Changes in interstitial pressure and cross-sectional area of the cubital tunnel and of the ulnar nerve with flexion of the elbow. An experimental study in human cadavera*. J Bone Jt Surg 80:492-501. https://doi.org/10.2106/00004623-199804000-00005

19. Patel VV, Heidenreich FPJ, Bindra RR et al (1998) Morphologic changes in the ulnar nerve at the elbow with flexion and extension: a magnetic resonance imaging study with 3-dimensional reconstruction. J Shoulder Elbow Surg 7:368-374. https://doi.org/10. 1016/s1058-2746(98)90025-8 
20. Lee Dellon A, Chang E, Henk Coert J, Campbell KR (1994) Intraneural ulnar nerve pressure changes related to operative techniques for cubital tunnel decompression. J Hand Surg 19:923-930. https://doi.org/10.1016/0363-5023(94)90091-4

21. Kim YS, Yeh LR, Trudell D, Resnick D (1998) MR imaging of the major nerves about the elbow: cadaveric study examining the effect of flexion and extension of the elbow and pronation and supination of the forearm. Skelet Radiol 27:419-426. https://doi. org/10.1007/s002560050411

22. Roodt T, van Dyk B, Jacobs S (2015) Ultrasound diagnosis of ulnar nerve entrapment by confirming baseline cross-sectional area measurement for normal and abnormal nerves. S Afr J Radiol. https://doi.org/10.4102/sajr.v19i1.747

23. Tandon A, Khullar T, Maheshwari S et al (2020) High resolution ultrasound in subclinical diabetic neuropathy: a potential screening tool. Ultrasound. https://doi.org/10.1177/1742271X20958034

24. Van BEW, Bathala L, Visser LH (2014) Difference in normal values of median nerve cross-sectional area between Dutch and Indian subjects. Muscle Nerve 50:129-132. https://doi.org/10. 1002 /mus. 24124

25. Hobson-Webb LD, Padua L (2009) Median nerve ultrasonography in carpal tunnel syndrome: findings from two laboratories. Muscle Nerve 40:94-97. https://doi.org/10.1002/mus.21286

26. Cartwright MS, Mayans DR, Gillson NA et al (2013) Nerve crosssectional area in extremes of age. Muscle Nerve 47:890-893. https://doi.org/10.1002/mus.23718
27. Cartwright MS, Shin HW, Passmore LV, Walker FO (2007) Ultrasonographic findings of the normal ulnar nerve in adults. Arch Phys Med Rehabil 88:394-396. https://doi.org/10.1016/j.apmr. 2006.12.020

28. Lothet EH, Bishop TJ, Walker FO, Cartwright MS (2019) Ultrasound-derived nerve cross-sectional area in extremes of height and weight. J Neuroimaging 29:406-409. https://doi.org/10.1111/jon. 12590

29. Kang S, Kim SH, Yang SN, Yoon JS (2016) Sonographic features of peripheral nerves at multiple sites in patients with diabetic polyneuropathy. J Diabetes Complicat 30:518-523. https://doi. org/10.1016/j.jdiacomp.2015.12.008

30. Chen J, Wang C-L, Wu S et al (2017) The feasibility of using high-resolution ultrasonography to assess ulnar nerve in patients with diabetes mellitus. J Ultrasonogr 17:160-166. https://doi.org/ 10.15557/JoU.2017.0024

31. Singh Y, Dixit R, Singh S et al (2019) High resolution ultrasonography of peripheral nerves in diabetic peripheral neuropathy. Neurol India 67:S71-S76. https://doi.org/10.4103/0028-3886.250719

Publisher's Note Springer Nature remains neutral with regard to jurisdictional claims in published maps and institutional affiliations. 